\title{
The experimental study of repairing cutaneous deficiency by transplanting the compound of mesenchymal stem cells and PLGA porous foam scaffolds
}

\author{
Gongxian Wang ${ }^{1}$, Yang Wang ${ }^{1}$, Weipeng Liu ${ }^{1}$, Yian Zhan ${ }^{1}$ \\ ${ }^{1}$ Department of Urology, The First Affiliated Hospital of Nanchang University, Nanchang, China
}

Our purposeis to evaluate the feasibility of regenerating or repairing damaged skin utilizing mesenchymol stem cells (MSCs) by tissue engineering techniques. Mesenchymol stem cells were isolated from New Zealand rabit of 1 month, cultured with DMEM complete medium in vitro. MSCs of 3 5 passages were seeded on porous foam PLGA[poly(lactic-co-glycolic acid) PLGA] scaffolds after marked by (4,6-Diamidino-2-phenylindole) DAPI, and then observed by scanning electron microscope and fluorescence microscope. The skin of $2 \times 2 \mathrm{~cm}^{2}$ were cut from the back of rabbit, and then the compound of MSCs-PLGA and the porous foam PLGA scaffolds alone were tansplanted on it respectively. After operation, wound healing was observed, HE stain, Van-Gieson stain and immunocytochemistry of cytokeratin AE1/AE3 were performed to detect the transplants after 5 weeks. After 3 days of cultivation, most of the MSCs adhered the wall. At the 10th day of cultivation, cells grew confluently and then passaged. Both scanning electron microscope and fluorescence microscope showed that cells grew well on porous foam PLGA scaffolds, and the number of cells increased gradually. Animal experiment showed that with the degradation of biodegradable polymer scaffolds, the skin was gradually replaced by the compound of MSCs-PLGA, HE stain showed the structure of the new skin were as the same as the normal skin, the cells of blue Fluorescent nucleus could been found in fluorescence microscope and immunocytochemistry showed cytokeratin expressed in cutinized layer and hair follicle. But the skin repaired by porous foam PLGA scaffolds showed little hair follicle and thickening fibrosis scar. Mesenchymal stem cells can be successfully isolated and cultured by adhering to the culture plastic. No obvious cytotoxicity was observed in PLGA polymer and it is a good carrier for MSCs. The damaged skin can be repaired utilizing the compound of MSCs and PLGA polymer.

Keywords: tissue engineering, mesenchymal stem cells, PLGA, wound healing

Cell Research (2008) 18:s66. doi: 10.1038/cr.2008.156; published online 4 August 2008

Correspondence: Gong-xian Wang

E-mail: wanggx-mr@126.com. 